\title{
Wege zum ökologischen Ziel
}

\section{Angesichts der großen Handlungsdefizite ist es verwunderlich, das bislang für die Umweltpolitik noch immer kein konsistenter, umfassender Zielkatalog existiert. Die Autoren tragen die bisherigen Ansütze einer entsprechenden Zielsetzung zusammen, diskutieren ihre Konsequenzen und extrahieren daraus ein Entscheidungsverfahren für die umweltpolitische Zielfindung.}

$\mathrm{U}$ Von Peter Jakubowski, Stefan Kotte und Henning Tegner ge Voraussetzung für einen ökologisch und ökonomisch effizienten Umweltschutz. Um so mehr erstaunt es, daß die gegenwärtige Umweltpolitik ohne einen konsistenten, umfassenden Zielkata$\log$ auskommt. Weder die öffentliche Diskussion noch die Wissenschaft. konnten bisher wesentlich dazu beitragen, die Umweltpolitik aus ihrem Schwebezustand im zielfreien Raum zu befreien. Auch die Umweltökonomik hat sich einschlägiger Arbeiten bisher weitgehend enthalten, sondern sich vorrangig auf die Untersuchung anreizkonformer Instrumente (Abgaben, Zertifikate, Haftung) konzentriert.

Erst in der jüngeren Diskussion um die umweltpolitische Zielfindung wurden Vorschläge mit dem Anspruch auf Rationalität und Praktikabilität eingebracht:

- Das Konzept einer Dematerialisierung der entwickelten Volkswirtschaften um den „Faktor $10^{\prime \prime}$, vertreten von den Autoren des Wuppertal Instituts für Klima, Umwelt, Energie.

- Das Drei-Säulen-Konzept von Klemmer, das darauf abhebt, umweltpolitische Zielsetzungen eingehenden Kosten-Nutzen-Analysen zu unterziehen.

- Der Verfahrensvorschlag zur Erstellung eines nationalen Umweltplans von Jänicke mit einem Schwerpunkt auf dem politischen Prozeß.

- Das Mehrstufenverfahren des Sachverständigenrats für Umweltfragen, das die wissenschaftlich-kognitiven Aufgaben im Vorfeld der umweltpolitischen Zielfindung betont.

\section{Die Dematerialisierungs- strategie}

Als ein Versuch, auf die aktuelle Zielfindungsdiskussion Einfluß zu nehmen, sind die zahlreichen Veröffentlichungen aus dem Wuppertal Institut für Klima, Umwelt, Energie anzusehen.
Kurzgefaßt fordern die Autoren, den Materialdurchfluß durch das ökonomische System bis zum Jahr 2050 auf ein Zehntel des heutigen Niveaus zu begrenzen (1). Anders als die traditionell emissionsorientierte Umweltpolitik setzt die ökologische Strategie der Wuppertaler Autoren damit am Stoffinput des ökonomischen Systems an.

Zur Rechtfertigung einer inputorientierten Umweltpolitik berufen sie sich vor allem auf das Scheitern der praktizierten Umweltpolitik und der umweltökonomischen Forschung. Eine nähere Analyse zeigt jedoch, daß ihr Konzept zu den Empfehlungen der ,traditionellen Umweltökonomie" keine Alternative bietet. Die wichtigsten Kritikpunkte lauten:

- Falscher Ansatzpunkt: Umweltpolitik legitimiert sich über die Aufgabe, Schäden von Mensch und Umwelt abzuwenden. Schäden entstehen durch Emissionen und durch die direkte Zerstörung von Naturgütern. Von daher setzt die Inputorientierung systematisch falsch an (2).

- Effizienzblindheit: Die ökonomischen Anpassungskosten an eine Strategie des „Faktor 10 " werden von den Wuppertaler Autoren regelmäßig mißachtet, was ihnen unter anderem den Vorwurf der Effizienzblindheit eingebracht hat (3).

- Verfabrensblindheit: Unklar bleibt in den Wuppertaler Veröffentlichungen, in welchen Verfahrensabläufen umweltpolitische Ziele generiert werden sollen. Der Vorschlag, die Ziele in einem Dialog von Politik, Wirtschaft und Umweltverbänden festzulegen, kann nicht befriedigen, weil er zu korporatistischen Lösungen zu Lasten Dritter neigt.

\section{Das Drei-Säulen-Modell}

Das in der Enquete-Kommission „Schutz des Menschen und der Umwelt" entwickelte und insbesondere von Klemmer befürwortete DreiSäulen-Konzept berücksichtigt neben dem Ziel der Erhaltung des ökologischen Realkapitals auch die Ökonomie- und die Sozialverträglichkeit der Umweltpolitik (4). Während sich der Begriff der Sozialverträglichkeit einer Operationalisierung weitgehend entzieht, soll die Ökonomieverträglichkeit nach Auffassung von Klemmer vor allem über die Einholung von Kosten-Nutzen-Analysen (KNA) der Umweltpolitik sichergestellt werden.

Mit Hilfe einer KNA ist es theoretisch denkbar, die optimale Umweltqualität einer Region zu ermitteln. Aufgabe des Analytikers wäre es, alle materiellen sowie immateriellen Kosten- und Nutzenkomponenten einer bestimmten Umweltqualität zu erfassen. Der politische Entscheidungsträger hätte dann nur noch die Aufgabe, die Umweltqualität mit dem höchsten NutzenKosten-Überschuß durchzusetzen.

Leider kann aufgrund von methodischen Problemen und erheblichen Unsicherheiten bei der Bewertung von Umweltgïtern von der KNA nicht erwartet werden, das optimale Umweltschutzniveau eindeutig zu bestimmen. Vielmehr werden Methodenvielfalt, Unsicherheiten über ökologische und ökonomische Parameter sowie eine überbordende Komplexität des ökologisch-ökonomischen Systems zwangsläufig zu einer hohen Ergebnisvielfalt führen. Darüber hinaus sind die Ergebnisse der KNA von den axiomatischen Grundlagen (Annahmen) abhängig.

Ein weiteres Hemmnis für eine Anwendung der KNA in der umweltpolitischen Praxis besteht darin, daß sie dem hypothetischen Kompensationskriterium nach Kaldor/Hicks verhaftet ist. Kosten und Nutzen werden unabhängig vom Ort ihres Anfallens saldiert und eine Maßnahme wird dann für gesamtwirtschaftlich vorteilhaft erachtet, wenn sie einen positiven Nettonutzen aufweist. Eine einfache Saldierung ist nicht allein aufgrund von methodischen Problemen unzulässig. Zusätzlich besteht die Gefahr, daß bei einer derart gravierenden Entscheidung wie der über mittel- bis langfristig verbindliche Umweltziele Anpassungslasten einseitig alloziiert werden. Die optimistische Argumentation, daß sich bei einer Vielzahl politischer Entscheidungen Kosten und Nutzen mittelfristig gleichmäßig verteilen, ist dann nicht zutreffend - es fehlt an Entscheidungen vergleichbaren Ausmaßes, welche individuelle Verluste bei der Umweltzielsetzung kompensieren könnten.

Für die Analyse sollte daher darauf verzichtet werden, alle Kosten zu quantifizieren und zu saldieren, um möglichst wenig Informationen über 
die Verteilung der Anpassungslasten aufzugeben. Vielmehr sollten alle Anpassungskosten disaggregiert dargestellt werden. Die Haushalte als Träger aller Anpassungslasten werden so in die Lage versetzt, ihren persönlichen Beitrag zur Umweltzielerfüllung abzuschätzen. Dazu benötigen sie Informationen über mögliche Veränderungen in der relativen Preisstruktur und strukturelle Anpassungserfordernisse in umweltintensiven Branchen, z.B. in Form drohender Arbeitsplatzverluste.

Es wäre verfehlt, von der KNA die Bestimmung der optimalen Umweltqualität zu erwarten. Mit ihrer Hilfe läßt sich jedoch der Bereich abstecken, in dem das Optimum zu vermuten ist. Somit kommt ihr die Funktion zu, den Suchraum für Umweltziele einzuschränken und die Informationsgrundlagen der Entscheidung zu verbessern. Dazu wird es in der Praxis erforderlich sein, mehrere Gutachter, nach Möglichkeit aus unterschiedlichen methodischen Schulen, zu Rate zu ziehen.

\section{- Nationale Umweltplanung im politischen System}

In einer Untersuchung für die Enquete-Kommission "Schutz des Menschen und der Umwelt" wurde von Jänicke/Carius/Jörgens der Prozeß der Erstellung eines nationalen Umweltplans in fünf OECD- Ländern untersucht (5). Auf Basis dieser Erkenntnisse wurden daraufhin Empfehlungen für Deutschland abgeleitet.

Zentrale Elemente des vorgeschlagenen Prozesses sind

- die Einrichtung einer speziellen Planungsinstitution („Rat für Umweltplanung“), die für die Beratung, Vorabklärung und Verabschiedung des Planes verantwortlich ist. Während das Plenum des Rates mit Vertretern von Bundesverwaltungen, Bundestag, Bundesländern, Verbänden und Wissenschaft eine beratende Funktion ausübt, werden in seinen Arbeitsgruppen Konsensgespräche mit wichtigen Zielgruppen geführt;

- wissenschaftliche Darstellung zentraler Umweltprobleme durch UBA, BMU und Forschungsinstitute sowie Abgabe von Zielempfehlungen;

- dezentrale Vereinbarungen und Selbstverpflichtungen von Ländern und Kommumen bzw. Branchen und Unternehmen.

Die wichtigsten Kritikpunkte an diesem Vorschlag lauten:
- Es ist zu bezweifeln, daß es in Konsensgesprächen zu einer Einigung über Umweltziele kommt. Kennzeichnend für die umweltpolitische Zielfindung ist evalutiver Dissens. Wenn überhaupt ein Konsens erreicht werden sollte, so dürfte dieser aufgrund divergierender Interessenlagen äußerst brüchig sein.

- Im Planerstellungsprozeß fehlt eine Abwägung von Kosten und Nutzen alternativer Ziele.

- Es kann durch den Planerstellungsprozess nicht sichergestellt werden, daß die Interessen aller potentiell Betroffenen adäquat berücksichtigt werden. Die Wähler können einerseits ihre Präferenzen nicht direkt zum Ausdruck bringen können, sondern treffen ihre Entscheidung anhand von Abstimmungsbündeln, die von den Parteien bzw. Repräsentanten dargestellt werden. Auf der anderen Seite kann nicht ausgeschlossen werden, daß sich der „Rat für Umweltplanung“ auf Kompromisse zu Lasten Dritter einläßt. Die politischen Akteure unterliegen dem Streben nach Wiederwahl, was ein Setzen langfristiger Umweltziele behindern dürfte. Zudem werden insbesondere die beteiligten Wirtschaftsverbände versuchen, die zur Diskussion gestellten Ziele zu verwässern (2).

\section{Mehrstufenverfahren}

Als Beitrag zur Diskussion um die Festsetzung von Umweltstandards hat der Rat von Sachverständigen für Umweltfragen (SRU) ein mehrstufiges Modell entwickelt (6). Der Rat sieht die naturwissenschaftliche Zustandsanalyse, die technischen Reduktionspotentiale und deren ökonomische Kosten, gesellschaftliche Einflußgrößen und politische Handlungsspielräume als tragende Säulen bei der Setzung von Umwelistandards an.

Das vorgeschlagene Mehrstufenverfahren setzt sich im Detail aus elf Verfahrensstufen zusammen. Zunächst ist ein Schutzobjekt zu definieren. Für die ermittelten Schutzobjekte ist sodann ein allgemeines Schutzniveau festzulegen: entweder als vollständiger oder als teilweiser Schutz, dessen Konkretisierung einer KostenNutzen-Abwägung bedarf. In den Schritten 3 bis 5 des Verfahrens erfolgt die naturwissenschaftliche Zustandsanalyse, die in einem naturwissenschaftlich orientierten Vorschlag für einen Standard mündet. Danach wird eine systematische Erarbeitung technischer Möglichkeiten zur Belastungsreduzierung vorgeschlagen. Auf der siebten Verfahrensstufe sollen alternative Optionen einer Kosten-Nutzen-Analyse unterzogen
Zum Thema ...

Sechsmal im Jahr: Die Fachzeitschrift für Umweltrecht und damit sechsmal im Jahr ein kompletter Überblick über das Umweltrecht.

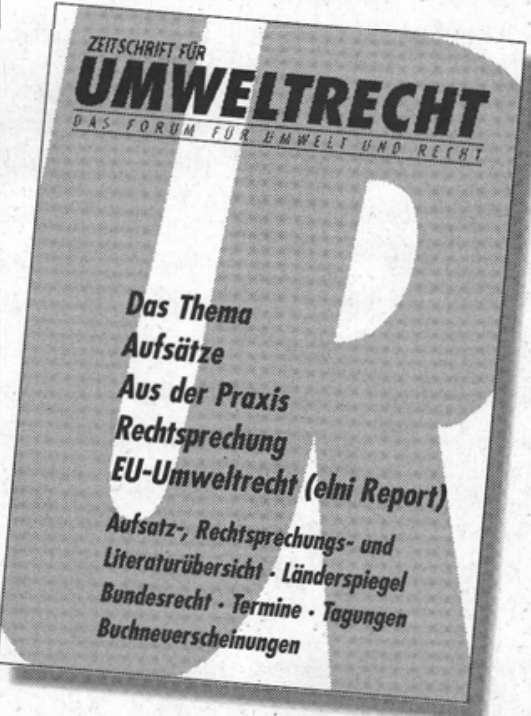

\section{Aktuelle wissenschaftliche Beiträge und Analysen}

- diskutieren Stand und Entwicklung des Umweltrechts.

\section{Ein umfangreicher Service-Teil}

- bringt die neueste Rechtsprechung,

- informiert über die aktuelle Gesetzesentwicklung auf Landes-, Bundes- und Europaebene und

- dokumentiert Aufsätze aus über einhundert Fachzeitschriften sowie wichtige Nachrichten, Termine und Buchneuerscheinungen.

Zu bestellen bei: Rhombos-Verlag

Kurfürstenstr. 17, 10785 Berlin Tel. 030/2619461, Fax 030/2616854 
werden, so daß auf der Stufe 8 ein gesellschaftlicher Diskurs eröffnet werden kann (Diskussionsphase). Es schließen sich die Entscheidungsphase, die Durchführung sowie Kontrollen und Fortschreibungen an.

Grundsätzlich ist anzumerken, daß das Verfahren eher für nachgeordnete Umweltstandards als für allgemeine, grundlegende Umweltziele konzipiert ist. Umweltstandards konkretisieren unbestimmte Rechtsbegriffe in Form von Grenzund Richtwerten und sind daher systematisch eher auf der Instrumental- als auf der Zielebene angesetzt.

Dem Anliegen, im Prozeß der Standardfindung eine möglichst breite gesellschaftliche Akzep$\operatorname{tanz}$ zu finden, soll dadurch Rechnung getragen werden, daß der Diskussion der verschiedenen gesellschaftlichen Gruppen ein breiter Raum zugebilligt wird. Es bleibt allerdings ungeklärt, wie nicht-organisierte Gruppen am Zielfindungsprozeß partizipieren können.

\section{Eine umweltökonomische Perspektive}

Wie aus der Kritik an den jeweiligen Zielfindungsstrategien deutlich wird, muß sich die umweltpolitische Zielfindung in einem Mehrstufenverfahren bewegen. Gefragt ist ein eklektischer Ansatz, der die Informationsgewinnungs-, Praktikabilitäts- und Rationalitätsvorteile der einzelnen Vorschläge kombiniert (2). Dabei dürfte evident sein, daß es zum Postulat nach ökonomischer Effizienz keine ernsthafte Alternative gibt.

Da umweltpolitische Zielentscheidungen Grundsatzcharakter haben und sich allokative wie distributive Entscheidungen darin vermengen, ist ein Verfahren zu suchen, daß bei geringeren Entscheidungsfindungskosten die von Buchanan/Tullock geforderte Einstimmigkeitsregel simuliert (7). Da der umweltpolitischen Zielsetzung ein erhebliches Konfliktpotential innewohnt, kann die Legitimität einer Entscheidung

... für denkende Anleger(innen) ...
Sie wollen Ihr Geld rentabel und
ökologisch sinnvoll anlegen?

Der Informationsdienst ÖKO-INVEST liefert Ihnen dazu alle zwei Wochen aktuelle und umfassende Informationen, z.B. über alternative Banken, aussichtsreiche ,grüne“ Aktien oder ökologische Beteiligungsmöglichkeiten in Investmentfonds bzw. nicht börsennotierten Gesellschaften. Damit Sie wissen, welche Unternehmen sauber sind und es auch bleiben.

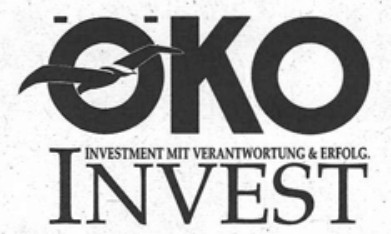

Ihr kostenloses Probeexemplar oder Infos zum Jahrbuch „Grünes Geld“ können Sie per Postkarte oder Fax abrufen bei der

\section{ÖKO-INVEST Verlags-GmbH \\ Schweizertalstr. 8-10/5 \\ A-1130 Wien \\ Tel. 0043/1/535 1815 \\ Fax 0043/1/535 4669 .} letztlich nur über ein Entscheidungsverfahren erreicht werden. Besteht ein Konsens

Diskussion dieser Zielbandbreiten im politischen Raum werden die Entscheidungsalternativen schließlich in einer allgemeinen Abstimmung den Stimmbürgern zur Wahl vorgelegt.

Wesentliche Charakteristika des vorgeschlagenen Zielfindungsverfahrens bestehen darin, daß die Rolle der Naturwissenschaft auf eine negative Zielsetzungskompetenz beschränkt wird, daß Nutzen-Kosten-Analysen den Suchraum für die Ziele abstecken sollen und die Entscheidung über zukünftige Umweltzustände von den eigentlich Betroffenen, also den Stimmbürgern, getroffen wird. Mit Hilfe dieser Verfahrenselemente ist eine Orientierung am erforderlichen Konsens offensichtlich eher sichergestellt als bei der einfachen Verabschiedung eines nationalen Umweltplans durch einen „,Rat für Umweltplanung" oder einer schlichten Festlegung auf eine Dematerialisierung der Volkswirtschaft um den Faktor 10.

\section{Literatur}

(1) Hinterberger, F.; Luks, F.; Stewen, M.: Ökologische Wirtschaftspolitik. Zwischen Ökodiktatur und Umweltkatastrophe, Berlin u.a.0. 1996.

(2) Jakubowski, P.; Tegner, H.; Kotte, S.: Strategien umweltpolitischer Zielfindung. Eine ökonomische Perspektive, Münster, Hamburg 1997.

(3) Gawel, E.: Neoklassische Umweltökonomie in der Krise, in: Köhn, J.; Welfens, M.J. (Hrsg.): Neve Ansötze in der Umweltökonomie, Marburg. 1996, S. 45-87.

(4) Klemmer, P.: Das Prinzip der Nachhaltigkeit - nevere stoffpolitische Ansätze, in: List-Forum für Wirtschafts- und Finanzpolitik 22 (1996), S. 313-330.

(5) Jänicke, M.; Carius, A.; Jörgens, H.: Konsensuale Zielbildung, Nationale Umweltplanung als never Ansatz in der Umweltpolitik, in: Ökologisches Wirtschaften 2 (1997), S. 6-7.

(6) Rat von Sachverständigen für Umwelffragen: Ein Mehrstufenmodell zur Festlegung von Umweltstandards, Zur Umsetzung einer dauerhaft-umweltgerechten Entwicklung, in: Zeitschrift für angewandte Umwelfforschung 9 (1996), S. 166-172.

(7) Buchanan, J.M.; Tullock, G.: The Calculus of Consent, Logical Foundations of Constitutional Democracy, 3. Aufl., Ann Arbor 1969.

weiteren Schritt werden

Zielbandbreiten von Naturwissenschaftlern und Medizinern vorgeschlagen, für die im Anschluß Kosten-Nutzen-Analysen erstellt werden. Nach einer

\section{Die Autoren}

Die Autoren
Dipl. Volkswirt Peter Jakubowski, Cand. rer. pol. Ste-
fan Kotte und Dipl. Volkswirt Henning Tegner sind
wissenschaftliche Mitarbeiter am Institut für Ver-
kehrswissenschaft der Universität Münster.
Kontakt: IVM, Am Stadtgraben 9, 48143 Münster
Tel::(0251) 83-22990, Fax: 83-28395
E-mail: 10ulwa@wiwi.uni-muenster.de


(c) 20I0 Authors; licensee IÖW and oekom verlag. This is an article distributed under the terms of the Creative Commons Attribution Non-Commercial No Derivates License (http://creativecommons.org/licenses/by-nc-nd/3.o/), which permits unrestricted use, distribution, and reproduction in any medium, provided the original work is properly cited. 